\title{
Análise dos potenciais medicinais do cajueiro (Anacardium occidentale Linn): uma
}

\section{breve revisão}

\author{
Analysis of the medicinal potentials of cashew tree (Anacardium occidentale Linn): a brief review \\ Análisis de los potenciales medicinales del anacardo (Anacardium occidentale Linn): una revisión
}

breve

Recebido: 09/01/2021 | Revisado: 18/01/2021 | Aceito: 18/01/2021 | Publicado: 21/01/2021

Thiago Emanuel Rodrigues Novaes
ORCID: https://orcid.org/0000-0002-3214-3220
Universidade Federal da Fronteira Sul, Brasil
E-mail: thiago.emanuel_rodri@ hotmail.com
Ana Selia Rodrigues Novaes
ORCID: https://orcid.org/0000-0002-9193-580X
Instituto Federal de Educação, Ciência e Tecnologia do Sertão Pernambucano, Brasil
E-mail: anaseliarn@ @otmail.com

\begin{abstract}
Resumo
A Anacardium occidentale Linn, conhecida popularmente como cajueiro, é uma espécie vegetal nativa do nordeste do Brasil. Dentre seus usos, pode-se destacar o medicinal, em processos terapêuticos e curativos. O objetivo deste estudo é discorrer acerca dos potenciais medicinais do cajueiro na medicina popular nordestina, analisando suas propriedades e principais usos pela população. Trata-se de uma breve revisão de literatura que se utilizou de estudos publicados entre 2002 e 2020, disponíveis na base de dados Google Acadêmico, as buscas se fizeram a partir das palavras-chave "Anacardium occidentale L.", "Anacardium occidentale L. caatinga", "Anacardium occidentale L. etnobotanica" e "Anacardium occidentale L. medicinal". Dos 30 trabalhos selecionados, 10 foram eleitos segundo critérios de inclusão para comporem o presente artigo. Os estudos indicam potenciais para tratamento e/ou cura de ferimentos, inflamações, infecções e problemas gastrointestinais, principalmente, em razão de propriedades derivadas de seus metabólitos secundários. Além disso, evidencia-se a necessidade da realização de mais estudos voltados à temática, visando o fortalecimento das pesquisas etnobotânicas do Brasil.
\end{abstract}

Palavras-chave: Etnobotânica; Anacardium occidentale; Medicina tradicional.

\begin{abstract}
Anacardium occidentale Linn, popularly known as cashew tree, is a plant species native to northeastern Brazil. Among its uses, medicinal can be highlighted in therapeutic and curative processes. The aim of this study is to discuss the medicinal potentials of cashew tree in popular medicine in the Northeast, analyzing its properties and main uses by the population. This is a brief review that used studies published between 2002 and 2020, available in the Google Scholar database, searches were made using the keywords "Anacardium occidentale L.", “Anacardium occidentale L. caatinga", "Anacardium occidentale L. etnobotânica" and "Anacardium occidentale L. medicinal". Of the 30 selected works, 10 were elected according to inclusion criteria to compose the present article. Studies indicate potentials for treating and / or curing wounds, inflammations, infections and gastrointestinal problems, mainly due to properties derived from their secondary metabolites. In addition, there is a need for further studies focused on the theme, aiming at strengthening ethnobotanical research in Brazil.
\end{abstract}

Keywords: Ethnobotany; Anacardium occidentale; Traditional medicine.

\section{Resumen}

Anacardium occidentale Linn, conocido popularmente como anacardo, es una especie vegetal originaria del noreste de Brasil. Entre sus usos, se puede destacar el medicinal en procesos terapéuticos y curativos. El objetivo de este estudio es discutir las potencialidades medicinales del anacardo en la medicina popular del Nordeste, analizando sus propiedades y principales usos por parte de la población. Es una revisión breve de la literatura que utilizó estudios publicados entre 2002 y 2020, disponibles en la base de datos Google Scholar, las búsquedas se realizaron utilizando las palabras clave "Anacardium occidentale L.", "Anacardium occidentale L. caatinga", "Anacardium occidentale L. etnobotânica" y "Anacardium occidentale L. medicinal ”. De las 30 obras seleccionadas, 10 fueron elegidas según criterios de inclusión para componer el presente artículo. Los estudios indican potenciales para tratar y/o curar heridas, inflamaciones, infecciones y problemas gastrointestinales, principalmente debido a propiedades derivadas de sus metabolitos secundarios. Además, es necesario realizar más estudios centrados en el tema, con el objetivo de fortalecer la investigación etnobotánica en Brasil.

Palabras clave: Etnobotánica; Anacardium occidentale; Medicina tradicional. 


\section{Introdução}

A etnobotânica pode ser compreendida como o estudo das sociedades humanas, passadas e presentes, atreladas aos conhecimentos ecológicos, evolucionários e simbólicos, reconhecendo a dinâmica existente das relações entre o ser humano e as plantas. $\mathrm{O}$ uso das espécies vegetais com finalidades terapêuticas e curativas remete à pré-história. No Brasil, isso se deve à forte influência cultural dos povos indígenas locais miscigenada às tradições africanas e europeias nos tempos de colonização do território (Alexiades, 1996; Almeida, 2003; Maioli-Azevedo \& Fonseca-Kruel, 2007).

No semiárido nordestino, as espécies vegetais nativas são amplamente utilizadas pelas comunidades locais, na medicina popular. Essas comunidades possuem uma farmacopéia natural ampla, em que parte dela é proveniente dos recursos vegetais encontrados nos ambientes naturais ocupados por estas populações, ou ainda cultivados em ambientes antropicamente alterados (Gomes et al., 2008).

A Anacardium occidentale Linn, conhecida popularmente como cajueiro (Figura 1), cajueiro branco, cajueiro roxo ou cajueiro comum, é uma espécie vegetal nativa do nordeste brasileiro que é amplamente aproveitada pela população, uma vez que, do cajueiro, pode-se fazer uso de muitas partes da planta com diversas finalidades, sejam elas medicinais ou alimentares. O pedúnculo, seu pseudofruto, é chamado de caju (Figura 2), e apresenta coloração variada em tons de amarelo a vermelho. Junto a ele, tem-se a amêndoa da castanha de caju que, em seu interior, armazena a castanha (Figura 3). O caju e a castanha são apreciados na culinária nordestina, tanto in natura quanto em outros produtos alimentícios.

Figura 1. Cajueiros (A. occidentale) no interior de Pernambuco.

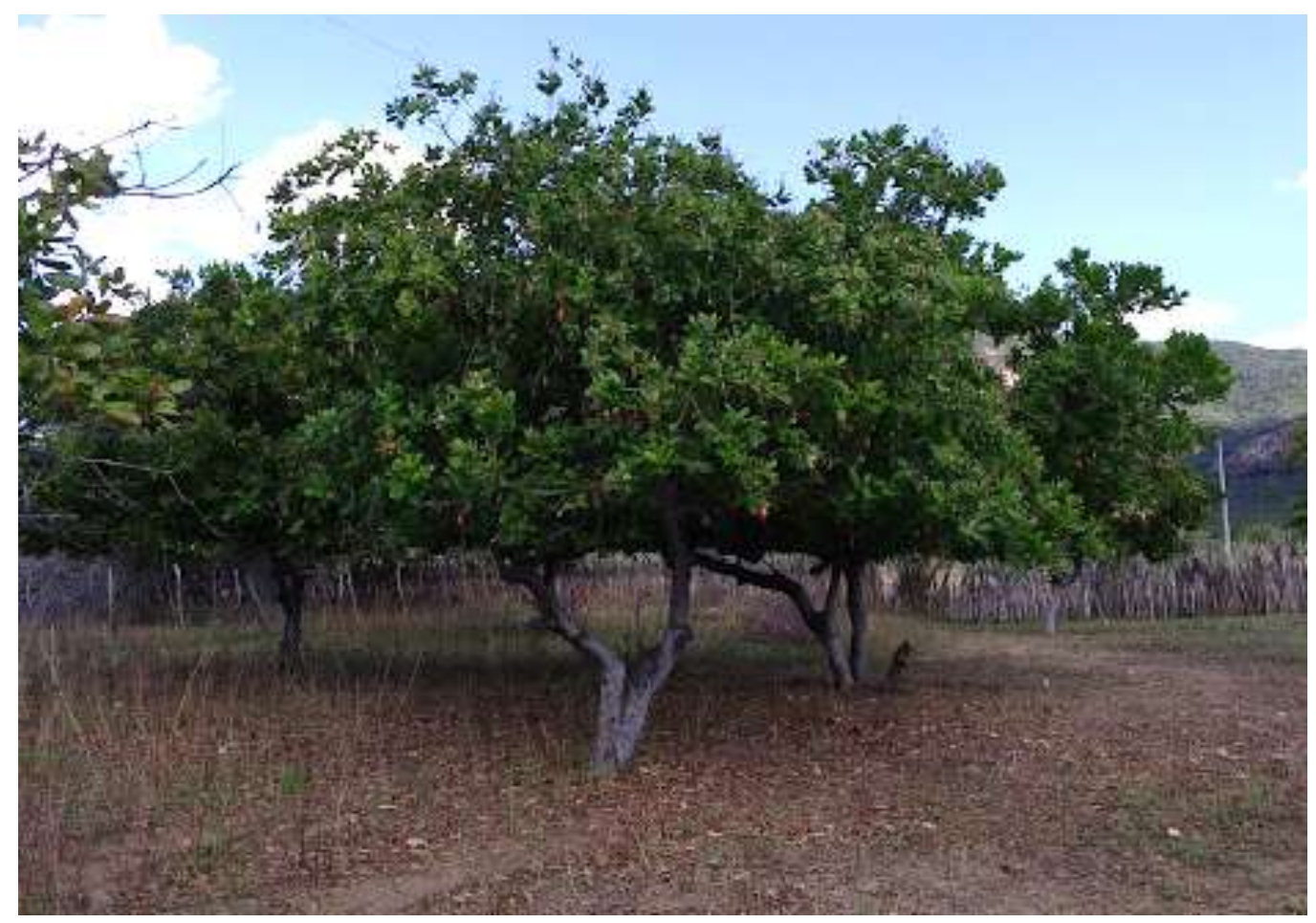

Fonte: Autores (2020). 
Figura 2. Cajus e amêndoas das castanhas de caju.

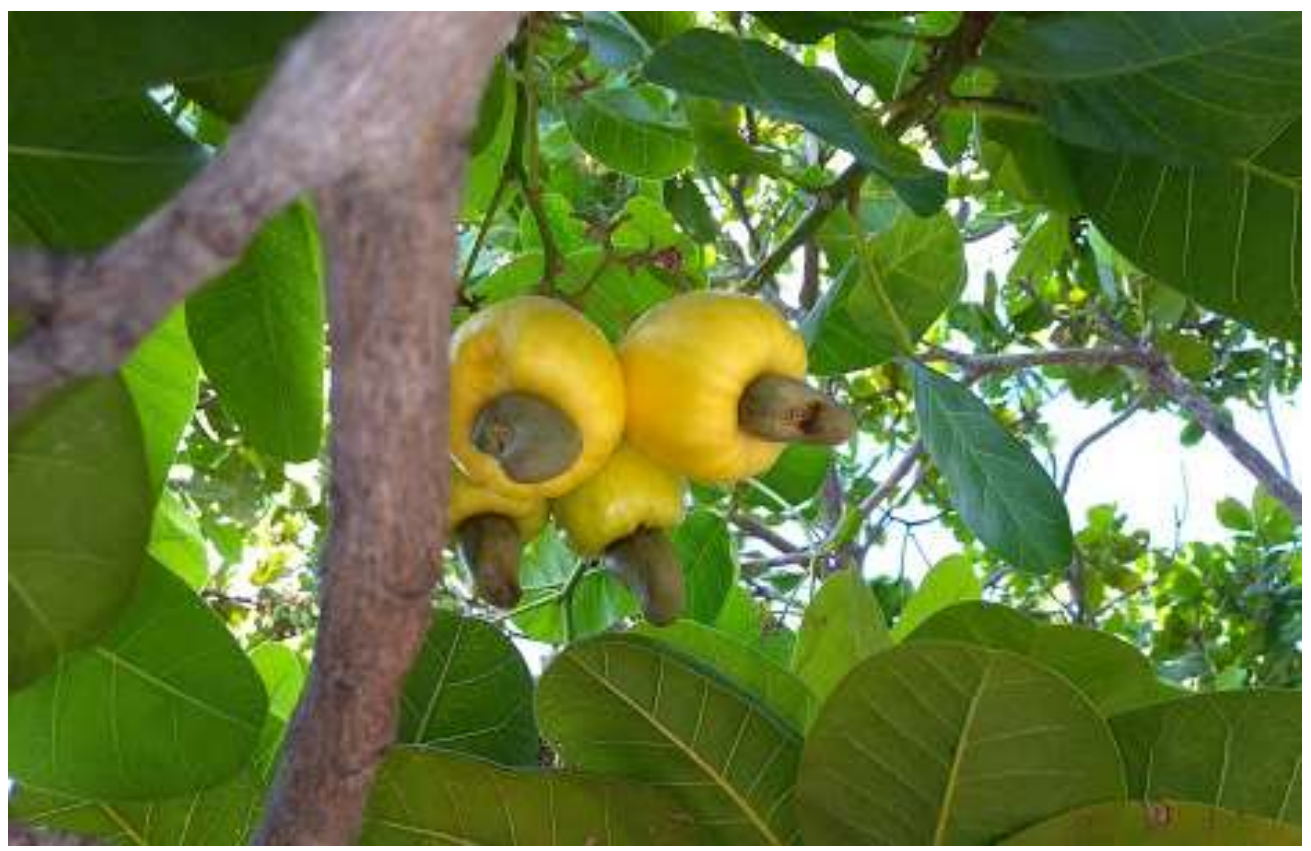

Fonte: Autores (2020).

Figura 3. Castanhas em pote. Pode-se observar as amêndoas queimadas no chão, após processo de retirada das castanhas.

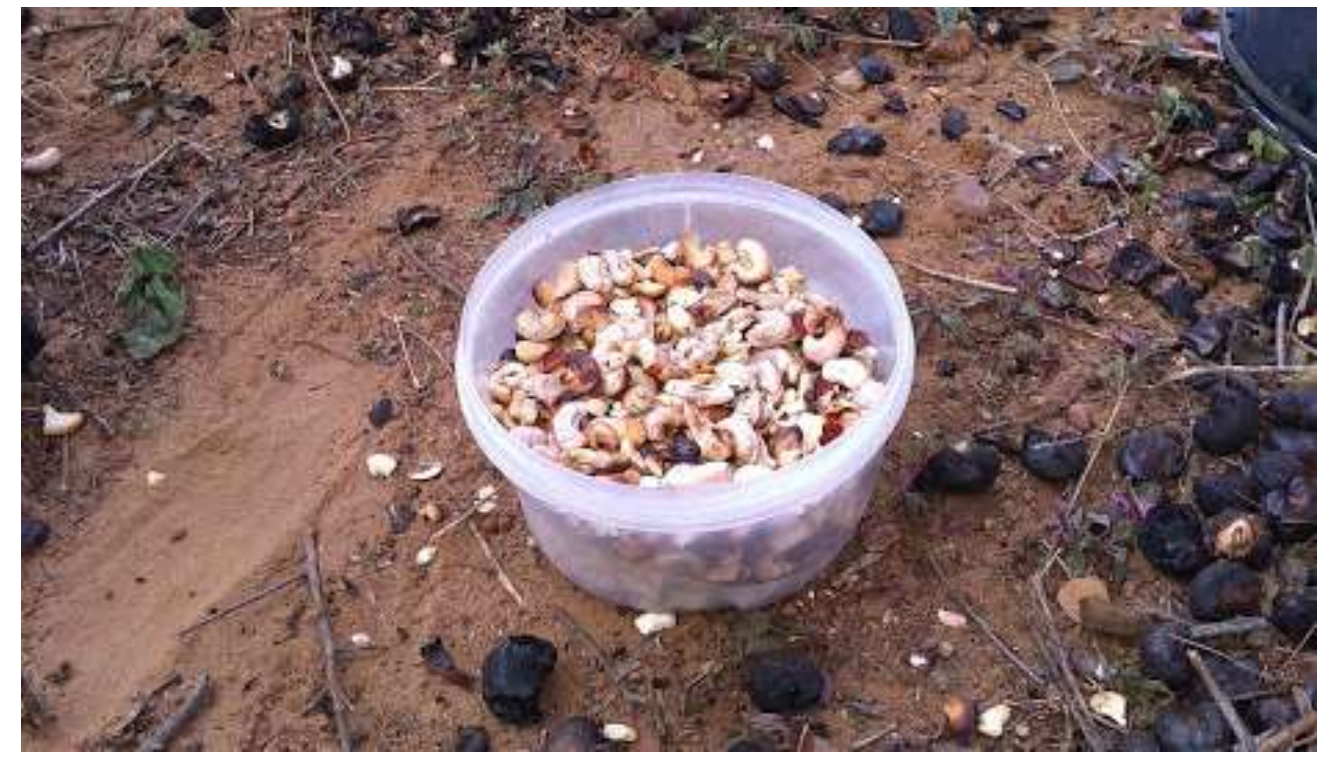

Fonte: Autores (2020).

Nessa perspectiva, o presente trabalho objetiva discorrer acerca dos potenciais medicinais do cajueiro (A. occidentale) na medicina popular nordestina, analisando suas propriedades e principais usos pela população.

\section{Metodologia}

Trata-se de uma breve revisão de literatura, em que foram selecionados estudos na base de dados Google Acadêmico, que após critérios de elegibilidade, foram incluídos na presente pesquisa. A busca utilizou as palavras-chave "Anacardium occidentale L.", “Anacardium occidentale L. caatinga”, “Anacardium occidentale L. etnobotanica” e "Anacardium occidentale L. medicinal". Foram incluídos artigos e trabalhos científicos de outros desenhos metodológicos, como tese de doutorado, 
publicados entre 2002 a 2020. O período da busca dos trabalhos ocorreu entre os dias 2 janeiro de 2021 e 9 de janeiro de 2021.

A seleção dos estudos para compor esta revisão foi feita por dois avaliadores independentes. Cada trabalho foi lido na íntegra e as informações foram organizadas em planilha, onde foram dispostos autoria e ano de publicação, objetivo, metodologia, resultados e conclusão.

A busca inicial selecionou 30 trabalhos. Destes, 2 dos estudos foram excluídos por duplicidade de algumas informações. Ademais, outros 18 foram excluídos por não se adequarem ao objetivo proposto neste trabalho. Ao final, foram incluídos 10 estudos para integrarem esta revisão.

Os critérios de elegibilidade dos trabalhos científicos para comporem este estudo incluíam relevância, ano de publicação, conteúdo, idioma, autores profissionais especializados em estudos etnobotânicos, tipo de estudo e objetividade das publicações, onde foram eleitos estudos em inglês e português, mais citados e relevantes na base de dados utilizada. Nesse sentido, foram excluídos desta revisão os estudos que não se adequavam ao objetivo proposto.

Ademais, os dados foram organizados em tabelas e discutidos na estrutura textual desta pesquisa. Além disso, utilizou-se de registros fotográficos de autoria própria para melhor identificação do cajueiro (A. occidentale).

\section{Resultados e Discussão}

A Tabela 1 apresenta os estudos eleitos incluídos na amostra final desta revisão e seus conteúdos, onde foram destacados objetivo, metodologia, resultados e conclusão.

Tabela 1. Estudos eleitos para a presente revisão sistemática.

\begin{tabular}{|c|c|c|c|c|}
\hline Autoria & Objetivo & Metodologia & Resultados & Conclusão \\
\hline $\begin{array}{l}\text { MOREIRA } \\
\text { et al. (2002) }\end{array}$ & $\begin{array}{c}\text { Analisar o resgate do } \\
\text { conhecimento de uma } \\
\text { comunidade no município de } \\
\text { Ilhéus (BA) sobre o uso de } \\
\text { plantas medicinais }\end{array}$ & $\begin{array}{l}\text { Levantamento de dados a } \\
\text { partir de entrevista com } \\
\text { moradores locais }\end{array}$ & $\begin{array}{l}\text { A análise final mostrou } \\
\text { a ocorrência de } 85 \\
\text { espécies de plantas } \\
\text { medicinais usadas na } \\
\text { forma de chá, xarope, } \\
\text { banho e outras formas }\end{array}$ & $\begin{array}{l}\text { A parte mais utilizada } \\
\text { das plantas foi a folha } \\
(64 \%) \text { seguida } \\
\text { daplanta toda }(13 \%), \\
\text { do fruto }(8 \%) \text {, da } \\
\text { casca }(7 \%) \text { e de outras } \\
\text { partes }(8 \%) \text { como } \\
\text { látex }\end{array}$ \\
\hline $\begin{array}{l}\text { MELO et } \\
\text { al. (2006) }\end{array}$ & $\begin{array}{l}\text { Determinar a atividade } \\
\text { antimicrobiana in vitro do } \\
\text { extrato da casca do caule de } \\
\text { A. occidentale L. em culturas } \\
\text { de Streptococcus } \mathrm{spp}\end{array}$ & $\begin{array}{l}\text { Estudo experimental com } \\
\text { extrato da casca do tronco } \\
\text { do cajueiro }\end{array}$ & $\begin{array}{l}\text { Os resultados sugerem } \\
\text { que o extrato tem } \\
\text { efeito sobre as espécies }\end{array}$ & $\begin{array}{c}\text { A pesquisa indica que } \\
\text { o extrato pode ser } \\
\text { usado } \\
\text { terapeuticamente na } \\
\text { odontologia como } \\
\text { agente antibacteriano }\end{array}$ \\
\hline $\begin{array}{l}\text { SILVA et } \\
\text { al. (2012) }\end{array}$ & $\begin{array}{l}\text { Avaliar o comportamento } \\
\text { reológico, além dos } \\
\text { parâmetros microbiológicos, } \\
\text { químicos e físico-químicos } \\
\text { das polpas de caju, acerola e } \\
\text { manga }\end{array}$ & $\begin{array}{l}\text { As medidas experimentais } \\
\text { foram realizadas em } \\
\text { reômetro rotacional de } \\
\text { cilindros concêntricos e os } \\
\text { dados experimentais foram } \\
\text { ajustados aos modelos de } \\
\text { Ostwald-de-Waelle e } \\
\text { Bingham }\end{array}$ & $\begin{array}{c}\text { As amostras } \\
\text { apresentaram } \\
\text { comportamento não- } \\
\text { newtoniano e caráter } \\
\text { pseudoplástico }\end{array}$ & $\begin{array}{l}\text { Riqueza nutricional } \\
\text { dos frutos estudados }\end{array}$ \\
\hline $\begin{array}{c}\text { SILVA } \\
(2012)\end{array}$ & $\begin{array}{l}\text { Avaliar a ação antioxidante e } \\
\text { antimicrobiana e investigar a } \\
\text { composição química dos } \\
\text { extratos das flores, folhas e } \\
\text { da casca do caule do cajueiro }\end{array}$ & $\begin{array}{c}\text { Revisão de literatura } \\
\text { associada a estudo } \\
\text { experimental com testes em } \\
\text { micro-organismos }\end{array}$ & $\begin{array}{l}\text { O estudo indicou ação } \\
\text { antimicrobiana e } \\
\text { presença de } \\
\text { metabólitos } \\
\text { secundários nos } \\
\text { extratos }\end{array}$ & $\begin{array}{l}\text { As propriedades } \\
\text { antimicrobianas e } \\
\text { antioxidantes das } \\
\text { flores podem estar } \\
\text { relacionadas à } \\
\text { presença de } \\
\text { flavonoides e/ou de } \\
\text { compostos fenólicos }\end{array}$ \\
\hline SOUZA et & Avaliar utilização de plantas & Estudo descritivo, & A maioria dos & Dentre as espécies \\
\hline
\end{tabular}


al. (2013) medicinais com atividade antimicrobiana pelos usuários do Sistema Único de Saúde do município de Campina Grande (PB)

Levantamento etnobotânico RIBEIRO et das plantas medicinais no al. (2014) município de Assaré (CE)

ARAUJO et al. (2018)

FURTADO
et al. (2019)

PADILHA et al. (2020)

RIBEIRO et al. (2020)

\section{Apresentar uma visão geral das aplicações farmacológicas e \\ tecnológicas relacionadas à utilização da goma do cajueiro (GC)}

Avaliar o efeito associado entre o gel à base da casca do cajueiro (Anacardium occidentale L) e o ultrassom terapêutico sobre o processo de cicatrização de feridas cutâneas em camundongos Identificar dados disponíveis na literatura sobre as propriedades fitoterápicas de A. occidentale Apresentar um estudo bibliométrico das propriedades antimicrobianas e antifúngicas do caule e da folha do cajueiro frente a patógenos orais

\author{
transversal, com abordagem \\ quantitativa, cuja amostra \\ constituiu-se de 220 \\ usuários
} Entrevistas com observação
direta

Revisão de literatura

Estudo experimental com 24 camundongos, divididos em 4 grupos, os quais passaram por diferentes tempos experimentais

Revisão de literatura

Revisão de literatura e estudo bibliométrico indivíduos obtém

plantas medicinais no comércio local utilizando-as por indicação de familiares

Elevada riqueza da flora medicinal presente na caatinga

\section{Observou-se 4} atividades farmacológicas para a GC: cicatrizante, antibacteriana, gastroprotetora e antidiarreica

Melhora do processo inflamatório inicial e aceleração do reparo tecidual nos grupos tratados com o UST, extrato, assim como, através da sua interação

Encontrou-se 98 propriedades diferentes de A. occidentale com finalidades terapêuticas A maioria dos testes realizados mostrou potencial antifúngico positivo e propriedades antimicrobianas promissoras mais citadas, destacase a Anacardium occidentale $\mathrm{L}$.

Identificou notável conhecimento e/ou uso das propriedades terapêuticas das espécies vegetais O campo de investigação para as atividades farmacológicas da Goma do Cajueiro é bastante amploe necessita ser mais explorado

Resultados satisfatórios sobre o processo de reparação tecidual Os efeitos antioxidantes e antimicrobianos foram os principais relatados Eficácia no tratamento de doenças que acometem a cavidade oral, como a cárie, a candidose e infecções periapicais

Fonte: Autores (2021).

A Tabela 2 sistematiza os achados nos trabalhos científicos com caráter experimental, onde foram analisadas as propriedades da A. occidentale.

Tabela 2. Propriedades encontradas nos estudos experimentais.

\begin{tabular}{cccc}
\hline Autoria e ano & Parte usada & Compostos analisados & Atividades \\
\hline MELO et al. (2006) & Casca & Extrato & Antimicrobiana \\
SILVA (2012) & Casca, flores e folhas & Extratos e metabólitos secundários & Antimicrobianas e antioxidantes \\
SILVA et al. (2012) & Pseudofruto & Vitamina C, antocianinas e polifenóis & Antioxidante \\
FURTADO et al. (2019) & Casca & Extrato alcoólico & Cicatrizante \\
\hline
\end{tabular}

Fonte: Autores (2021).

A Tabela 3 integra as propriedades discorridas nos estudos de revisão com a A. occidentale. 
Tabela 3. Atividades terapêuticas da A. occidentale.

\begin{tabular}{cccc}
\hline Autoria e ano & $\begin{array}{c}\text { Parte da planta } \\
\text { utilizada }\end{array}$ & Forma de uso & Indicações terapêuticas \\
\hline MOREIRA et al. (2002) & Casca e folha & $\begin{array}{c}\text { Chá } \\
\text { Líquida (após } \\
\text { maceração) }\end{array}$ & Diarréia \\
SOUZA et al. (2013) & Casca & Infecções urinárias \\
RIBEIRO et al. (2014) & Casca e entrecasca & Decocção ou infusão & $\begin{array}{c}\text { Ferimentos e inflamações de ovário e } \\
\text { gerais }\end{array}$ \\
\hline
\end{tabular}

Fonte: Autores (2021).

A Tabela 4 dispõe os achados na literatura acerca das atividades terapêuticas do cajueiro pela população, apresentando partes da planta utilizadas, formas de uso e indicações.

Tabela 4. Propriedades medicinais discorridas nos estudos de revisão.

\begin{tabular}{|c|c|c|}
\hline $\begin{array}{c}\begin{array}{c}\text { Autoria e } \\
\text { ano }\end{array} \\
\end{array}$ & $\begin{array}{c}\text { Parte } \\
\text { utilizada }\end{array}$ & Potencialidades descritas \\
\hline $\begin{array}{l}\text { ARAUJO } \\
\text { et al. } \\
(2018)\end{array}$ & $\begin{array}{l}\text { Goma do } \\
\text { cajueiro }\end{array}$ & Cicatrizantes, antibacterianas, gastroprotetoras e antidiarreicas \\
\hline $\begin{array}{l}\text { PADILHA } \\
\text { et al. } \\
\text { (2020) }\end{array}$ & - & $\begin{array}{c}\text { Anti-inflamatória, antioxidante, antimicrobiana, antidiabética, cicatrizante, analgésica, efeitos no } \\
\text { aparelho digestório, no sistema nervoso autônomo, respiratório e sanguíneo, efeitos hipotensivos e } \\
\text { cardioinibitórios, protetor contra a disfunção sexual masculina, antimutagênico, antimetastático e } \\
\text { clastogênico e uso odontológico }\end{array}$ \\
\hline $\begin{array}{l}\text { RIBEIRO } \\
\text { et al. } \\
(2020)\end{array}$ & $\begin{array}{l}\text { Caule e } \\
\text { folhas }\end{array}$ & Atividades antimicrobianas e antifúngicas \\
\hline
\end{tabular}

Fonte: Autores (2021).

A A. occidentale é uma espécie nativa do nordeste brasileiro, distribuída amplamente pelo território em razão de possuir grande importância comercial, sendo encontrada principalmente em climas tropicais e subtropicais. Seu pseudofruto, o caju, é utilizado na produção de doces e iguarias. Na cultura nordestina, os extratos das folhas, das cascas, das raízes, assim como a castanha e a goma do cajueiro são bastante utilizados na medicina tradicional para o tratamento de inúmeras enfermidades (Araujo et al., 2018; Aragão, 2015; Agra, Freitas \& Barbosa-Filho, 2007).

Achados experimentais com extratos do cajueiro indicam a presença de substâncias, como elevada concentração de vitamina $\mathrm{C}$, antocianinas, polifenóis e outros metabólitos secundários com atividades antioxidantes e antimicrobianas, que facilitam a terapêutica de processos infecciosos, inflamatórios e cicatrizantes, principalmente (Furtado et al., 2019; Silva et al., 2012; Silva, 2012; Melo et al., 2006).

Os usos na medicina popular são múltiplos. Segundo relatos, nos estudos analisados, a casca, a entrecasca e as folhas são mencionadas como as partes utilizadas para a produção de chás sob indicações terapêuticas para tratamento e/ou cura de ferimentos, inflamações, infecções e problemas gastrointestinais (Ribeiro et al., 2014; Souza et al., 2013; Moreira et al., 2002). Ademais, é sabido que há outras formas de uso do cajueiro com essas finalidades, como lambedores, garrafadas e aplicação direta.

\section{Conclusão}

A análise da literatura evidenciou que o cajueiro (A. occidentale) apresenta grande potencial medicinal, em razão de reter metabólitos secundários responsáveis pelos efeitos descritos no decorrer deste estudo. Suas indicações terapêuticas, na medicina popular, representam riqueza de possibilidades para ações farmacológicas e tratamentos alternativos, que envolvem 
processos inflamatórios, infecciosos e cicatrizantes. Dessa forma, torna-se necessária a realização de mais estudos voltados à temática, visando o fortalecimento das pesquisas etnobotânicas no Brasil.

\section{Referências}

Agra, M. D. F., Freitas, P. F. D., \& Barbosa-Filho, J. M. (2007). Synopsis of the plants known as medicinal and poisonous in Northeast of Brazil. Revista Brasileira de Farmacognosia, 17(1), 114-140.

Alexiades, M. N., \& Sheldon, J. W. (1996). Selected guidelines for ethnobotanical research: a field manual (No. Sirsi) i9780893274047).

Almeida, M. Z. D. (2003). Plantas medicinais. Edufba.

Aragão, J. A. D. S. (2015). Análise e aplicações biotecnologias de proteínas ligantes à quitina de sementes de cajueiro anão precoce (Anacardium occidentale var. nanum).

Araujo, S., Sousa, I. J. O., Gonçalves, R. L. G., de Sousa França, A. R., dos Santos Negreiros, P., da Silva Brito, A. K., \& de Sousa Lima, E. B. (2018). Aplicações Farmacológicas e Tecnológicas da Goma do Cajueiro (Anacardium Occidentale L.) um Produto Obtido da Flora Brasileira. Revista GEINTECGestão, Inovação e Tecnologias, 8(1), 4292-4305.

Furtado, R. A. A., Noleto, M. L. P., Pessoa, D. R., da Silva Almeida, V., Maia Filho, A. L. M., Uchôa, V. T., \& dos Santos Alves, W. (2019). Ação do gel Anacardium Occidentale L. associado ao ultrassom terapêutico no processo de cicatrização em camundongos. Saúde (Santa Maria), 45 (2), 15.

Gomes, E. C., Barbosa, J., Vilar, F. C., Perez, J., Vilar, R., Graduando Engenharia, U. F. P. B., \& Dias, T. (2008). Plantas da caatinga de uso terapêutico: levantamento etnobotânico. Engenharia Ambiental: Pesquisa e Tecnologia, 5(2).

Maioli-Azevedo, V., \& Fonseca-Kruel, V. S. D. (2007). Plantas medicinais e ritualísticas vendidas em feiras livres no Município do Rio de Janeiro, RJ, Brasil: estudo de caso nas zonas Norte e Sul. Acta Botanica Brasilica, 21(2), 263-275.

Melo, A. F., Santos, E. J. V., Souza, L. F., Carvalho, A. D. A. T., Pereira, M. D. S. V., \& Higino, J. S. (2006). Atividade antimicrobiana in vitro do extrato de Anacardium occidentale L. sobre espécies de Streptococcus. Revista brasileira de farmacognosia, 16(2), 202-205.

Moreira, R. D. C. T., Costa, L. C. D. B., Costa, R. C. S., \& Rocha, E. A. (2002). Abordagem etnobotânica acerca do uso de plantas medicinais na Vila Cachoeira, Ilhéus, Bahia, Brasil. Acta farmacêutica bonaerense, 21(3), 205-2011.

Padilha, J. A., Vieira, L. N., Magalhães, V. F., Reginato, R. E. D., Lima, C. M. B. L., \& Diniz, M. D. F. F. M. (2020). Therapeutic effects of Anacardium occidentale: an integrative review. Acta Brasiliensis, 4(3), 178-186.

Ribeiro, A. D., Júnior, E. C. F., Júnior, J. G. R., Costa, B. P., Freire, J. C. P., de Souza Melo, W. O., \& Pereira, J. V. (2020). Potencial antimicrobiano do Anacardium occidentale Lin. contra patógenos orais. Research, Society and Development, 9(8), e883986459-e883986459.

Ribeiro, D. A., Macêdo, D. G., Oliveira, L. G. S., Saraiva, M. E., Oliveira, S. F., Souza, M. M. A., \& Menezes, I. R. A. (2014). Potencial terapêutico e uso de plantas medicinais em uma área de Caatinga no estado do Ceará, nordeste do Brasil. Revista Brasileira de Plantas Medicinais, 16(4), 912-930.

Silva, L. M. R., Maia, G. A., de Figueiredo, R. W., de Sousa, P. H. M., da Costa Gonzaga, M. L., \& de Figueiredo, E. A. T. (2012). Estudo do comportamento reológico de polpas de caju (Anacardium occidentale, L.), acerola (Malpighia emarginata, DC) e manga (Mangifera indica, L.). Semina: Ciências Agrárias, $33(1), 237-247$.

Silva, R. A. (2012). Antimicrobial Action Anacardium Occidentale L.: biotechnological potential in the generation of anti-caries products. 2012.154 f. Tese (Doutorado em Fertilização) - Universidade Federal do Maranhão, São Luís.

Souza, C. M. P., Brandão, D. O., Silva, M. S. P., Palmeira, A. C., Simões, M. O. S., \& Medeiros, A. C. D. (2013). Utilização de plantas medicinais com atividade antimicrobiana por usuários do serviço público de saúde em Campina Grande-Paraíba. Revista Brasileira de Plantas Medicinais, 15 (2), $188-193$. 